\title{
Characteristics and Perioperative Complications of Hip Fracture in the Elderly With Acute Ischemic Stroke: A Cross-Sectional Study
}

\section{Yaqian Zhang}

the Third Hospital of Hebei Medical University

Mingming Fu

the Third Hospital of Hebei Medical University

Junfei Guo

the Third Hospital of Hebei Medical University

Yuqi Zhao

the Third Hospital of Hebei Medical University

Zhiqian Wang ( $\nabla$ w18533112890@163.com )

the Third Hospital of Hebei Medical University

\section{Zhiyong Hou}

the Third Hospital of Hebei Medical University

\section{Research Article}

Keywords: Hip fracture, Ischemic stroke, Characteristics, Complications

Posted Date: January 31st, 2022

DOI: https://doi.org/10.21203/rs.3.rs-1293840/v1

License: (1) This work is licensed under a Creative Commons Attribution 4.0 International License.

Read Full License 


\section{Abstract}

Background: Patients with acute ischemic stroke (AIS) after hip fracture in the elderly have worse prognosis. We aimed to describe the characteristics and complications of hip fracture with AIS in the elderly.

Methods: This cross-sectional study selected patients with hip fracture (age $\geq 65$ years) from January 2018 to September 2020. The collected data included age, sex, fracture types, comorbidities. In above screened patients, we further collected cerebral infarction related information of AIS patients. Risk factors for AIS after hip fracture were identified.

Results: 60 patients (mean age 79.7 years; female 56.7\%) occurred AIS after hip fracture in 1577 cases. Among 60 AIS patients, the most common cause of fall was limb weakness or instability(46.7\%). The most common infarction type was $\mathrm{PACl}(70.0 \%)$. The majority of these infarction lesions were single (76.7\%) and most infarction lesions(65.0\%) were located in the left side. $81.7 \%$ of AIS patients had mild (NIHSS 0-4) AIS. Older patients with AIS after hip fracture were more frequently complicated by hypertension(73.3\%), prior stroke (46.7\%), diabetes(35.0\%) and were more likely to have hypoproteinemia(68.3\%), electrolyte disorders (66.7\%), anemia (65.0\%), deep vein thrombosis $(51.6 \%)$, pneumonia (46.6\%), cardiac complications (45.0\%). Combined with hypertension (OR $2.743,95 \% \mathrm{Cl} 1.471$ 5.115) and male sex(OR 1.946, 95\% $\mathrm{Cl} 1.146-3.304)$ were independent risk factors of AIS after hip fracture.

Conclusions: Older patients combined with hypertension are more likely to have AIS after hip fracture. For these patients, early preventions should be administered. AIS patients after hip fracture are prone to have multiple complications under traumatic stress, and we should enhance the management of these patients to reduce the stress and avoid occurrence of complications.

\section{Introduction}

As the accelerated rate of social aging, the number of older patients with hip fractures continually increase, which has become a global medical and health problem[1, 2]. There are approximately 1.5 million femoral fractures worldwide each year, and this number will rise to 6.3 million by 2050[3]. Hip fractures of the older adults, which are often referred to "the last fracture of life ", severely affect limb function and quality of life, and greatly increase social and family burdens[4]. A report showed that older patients have a high mortality of 8.4-36\% within one year after the fracture[5].

Previous studies have found that complications were a substantial contributor to high mortality in older patients with hip fracture[6, 7]. AIS is one of the catastrophic complications and associated with increased mortality in the hip fracture patients[8]. Fracture patients with perioperative AIS have worse functional recovery than those with non-AIS and require more care in the first year[9]. Currently, the existing studies focuses on the occurrence of stroke in the perioperative period and 1 year after the surgeries of hip fracture[10-12], while there are few studies on AIS after fracture. AIS after hip fracture 
makes patients unable to perform surgery in a short time and results in poor outcomes including impaired self-care, decreased quality of life and increased mortality[13]. Due to blood loss, pain and other factors, elderly patients with hip fracture are prone to traumatic stress reactions[14], which increase the risk of complications. We should pay more attention to these patients. However, there are few studies on the complications of AIS patients after fracture.

In this study, we retrospectively described characteristics and complications of hospitalized older adults with hip fractures who sustained AIS. These results will help people to understand characteristics of older hip fracture adults with AIS and provide treatment recommendations for these specific populations.

\section{Materials And Methods Patients and Groups}

In this retrospective study, we collected data in a single Level I trauma center in China from January 2018 to September 2020. The study protocol was approved by the institutional review board of the third Hospital of Hebei Medical University, and an exemption from the informed consent was obtained. Inclusion criteria were hip fracture patients aged 65 years or older and complete data available in medical records. The exclusion criteria were as follows: (1) had multiple fracture; (2) had pathological fracture, (3) had secondary fracture (4) incomplete medical information. Diagnosis of AIS should be based on consciousness, physical performance, head CT, and MRI performance. All AIS patients fulfilled the World Health Organization criteria for AIS [15]. Patients were retrospectively divided into 2 groups: those who developed AIS (AIS group) and those without AIS (No-AIS group).

\section{Data Collection}

The collected data of screened patients included sex, age, and fracture types, comorbidities in both groups. The incidence of AIS after hip fracture was 3.8\%. In AIS group, we further collected cerebral infarction related information. AIS was further classified into four subtypes as follows: total anterior circulation infarction (TACl), partial anterior circulation infarction (PACl), posterior circulation infarction (POCI), and lacunar infarction (LACI). Infarction lesions were classified into single infarction lesion and multiple infarction lesions. The sides of cerebral infarction included left side, right side or both sides. Severity of stroke was determined with the National Institutes of Health stroke scale (NIHSS) and categorized as mild (NIHSS <4), moderate (NIHSS 4-15), and severe (NIHSS > 15) stroke. According to the 10-year age interval, the AIS patients were divided into 3 groups: 65-74 years, 75-84 years, $\geq 85$ years.

Trauma mechanisms were classified as instability or fatigue, impacts injury, dizziness, slips and others.

\section{Statistical Analysis}

The Kolmogorov-Smirnov test was used to test the normality of continuous variables. Continuous parametric data was expressed as the mean \pm standard deviation and analyzed by student's t test or 
analysis of variance. Categorical variables were expressed as numbers and percentages and were compared using chi-squared test or Fisher's exact test. Univariate analysis was applied and variables with statistical significance were included in a multivariate model by stepwise logistic regression to identify independent risk factors of AIS. The $p$-value $<0.05$ was considered statistically significant and all analyzes were performed using IBM SPSS software (IBM Corp, Armonk, NY).

\section{Results}

\section{Age, Gender, Fracture types and Injury Mechanisms of AIS after Hip Fracture}

A total of 60 (34 female, 26male) older hip fracture patients occurred AIS in 1577cases. The incidence of AIS after hip fracture was $3.8 \%$ and incidence was higher in male than female(5.4\% vs $3.1 \%$ ).Among 60 (34 female, 26male) patients, the average age of patients was 79.7 years old (range 65-96), and 7584years account for the largest percentage (45\%). 27(45.0\%) suffered femoral neck fracture and $33(55.0 \%)$ suffered trochanteric fracture. The most common cause of fracture was instability or weakness, which was present in $46.7 \%$ of the patients. Other reasons included impact slips $(10,16.7 \%)$, dizziness $(10,16.7 \%)$, impact injury $(5,8.3 \%)$, and others $(7,11.6 \%)$. The specific information was presented in Table 1. 
Table 1

Age, gender, fracture types and injury mechanisms

\begin{tabular}{|lll|}
\hline acute ischemic stroke & Number (n) & Percentage(\%) \\
\hline Gender & & \\
\hline Female & 34 & 56.7 \\
\hline Male & 26 & 43.3 \\
\hline Age & & \\
\hline $65-74$ & 17 & 28.3 \\
\hline $75-84$ & 27 & 45.0 \\
\hline$\geq 85$ & 16 & 26.7 \\
\hline Fracture type & & \\
\hline Neck of femur & 27 & 45.0 \\
\hline Intertrochanteric & 33 & 55.0 \\
\hline Injury mechanism & & \\
\hline Instability or weakness & 28 & 46.7 \\
\hline Slips & 10 & 16.7 \\
\hline Dizziness & 10 & 16.7 \\
\hline Impact injury & 5 & 8.3 \\
\hline Other & 7 & 11.6 \\
\hline Notes: Values are presented as the number (n) and Percentage(\%) & \\
\hline & & \\
\hline
\end{tabular}

\section{Characteristics of AIS}

Table 2 showed that the types of AIS in elderly hip fracture patients with AIS were PACl $(42,70.0 \%), \mathrm{POCl}$ (9, 15.0\%), LACl (8, 13.3\%),TACl (1, 1.6\%).There were $46(76.7 \%)$ cases of solitary lesion and $14(23.3 \%)$ cases of multiple cerebral infarction and most of them were located on the left side(65\%). In addition, in these cases, there were 49(81.7\%), 9(15.0\%) and 2(3.3\%) patients with mild (NIHSS<4), moderate (NIHSS 4-15), and severe (NIHSS > 15) stroke, respectively. The specific information was presented in Table 2. 
Table 2

The characteristics of AIS

\begin{tabular}{|lll|}
\hline & Number ( $n$ ) & Percentage(\%) \\
\hline Type of cerebral infarction & & \\
\hline PACl & 42 & 70.0 \\
\hline POCl & 9 & 15.0 \\
\hline LACl & 8 & 13.0 \\
\hline TACl & 1 & 5.0 \\
\hline Distribution of cerebral infarction lesions & & \\
\hline Single infarction lesion & 46 & 76.7 \\
\hline multiple infarction lesion & 14 & 23.3 \\
\hline Side of cerebral infarction & & \\
\hline Left side & 39 & 65.0 \\
\hline Right side & 18 & 30.0 \\
\hline Both sides & 3 & 5.0 \\
\hline NIHSS score & & \\
\hline$<4$ & 49 & 81.7 \\
\hline $4-15$ & 9 & 15.0 \\
\hline$>15$ & 2 & 3.3 \\
\hline Notes: Values are presented as the number (n) and percentage(\%) & \\
\hline
\end{tabular}

\section{Comorbidities, Complications of AIS after Hip Fracture}

Among 60 patients, there were 20 patients combined with three or more comorbidities, accounting for $33.3 \%$. The most common comorbidity was hypertension, which was presented in $73.3 \%$ of the patients. Other common comorbidities included prior stroke (46.7\%), diabetes (35.0\%). 55(91.6\%) patients were presenting with at least one complication, which consisted of hypoproteinemia (68.3\%), electrolyte disorders (66.7\%), anemia (65.0\%), deep vein thrombosis (51.6\%), pneumonia (46.6\%), cardiac complications (45.0\%). The cardiac complications included heart failure (30.0\%), arrhythmia (16.6\%) and myocardial infarction (1.6\%).The specific information was presented in Table 3 and figure 1. 
Table 3

Comorbidities and Complications of AIS after hip fracture

\begin{tabular}{|lll|}
\hline & Number $(\mathbf{n})$ & Percentage(\%) \\
\hline Comorbidities $\mathbf{n}(\%)$ & & \\
\hline Hypertension & 44 & 73.3 \\
\hline Prior stroke & 28 & 46.7 \\
\hline Diabetes & 21 & 35.0 \\
\hline Coronary heart disease & 14 & 23.3 \\
\hline Arrhythmia & 5 & 8.3 \\
\hline Tumor & 4 & 6.7 \\
\hline Pulmonary disease & 4 & 6.7 \\
\hline Comorbidities $\geq 3$ & 20 & 33.3 \\
\hline Complications, $\mathrm{n}(\%)$ & & \\
\hline Total & 55 & 91.6 \\
\hline Hypoproteinemia & 41 & 68.3 \\
\hline Electrolyte disorders & 40 & 66.7 \\
\hline Anemia & 39 & 65.0 \\
\hline Deep vein thrombosis & 31 & 51.6 \\
\hline Pneumonia & 28 & 46.6 \\
\hline Cardiac complications & 27 & 45.0 \\
\hline Heart failure & 18 & 30.0 \\
\hline Arrhythmia & 10 & 60.0 \\
\hline Myocardial infarction & 1 & and percentage(\%) \\
\hline Complications $\geq 3$ & 24 & \\
\hline Notes: Values are presented as the number $(\mathrm{n})$ & & \\
\hline & & \\
\hline
\end{tabular}

\section{The Comparison of the AIS Group and No-AIS Group}

As shown in Table 4, among these 1577 patients, the average age was 79.7 years old (ranging from 65 to 104 years old). There were more female patients than male patients( $69.4 \%$ vs $30.6 \%)$. There was a higher proportion of male in the AIS group compared with the non-AIS group ( $43.3 \%$ vs $30.1 \%, p=0.029)$. AIS 
patients had more comorbidities(number $\geq 3, p=0.039)$, including hypertension $(p=0.000)$ and diabetes $(p=0.035)$. Other characteristics were similar between the two groups.

Table 4

The Comparison of the AIS group and No-AIS group

\begin{tabular}{|c|c|c|c|c|}
\hline & $\begin{array}{l}\text { Overall } \\
(n=1577)\end{array}$ & $\begin{array}{l}\text { AIS } \\
(n=60)\end{array}$ & $\begin{array}{l}\text { No-AIS } \\
(n=1517)\end{array}$ & $p$ Value \\
\hline \multicolumn{5}{|l|}{ Age (year) } \\
\hline mean years & $79.7 \pm 7.3$ & $79.8 \pm 6.9$ & $79.7 \pm 7.3$ & 0.217 \\
\hline \multicolumn{5}{|l|}{ Gender n (\%) } \\
\hline Male & 483凶30.6邓 & $26(43.3)$ & $457(30.1)$ & 0.029 \\
\hline Female & $1094 \llbracket 69.4 \rrbracket$ & $34(56.7)$ & $1060(69.9)$ & \\
\hline \multicolumn{5}{|l|}{ Fracture type n (\%) } \\
\hline Neck of femur & $758(48.1)$ & $27(45.0)$ & $731(48.2)$ & 0.628 \\
\hline Intertrochanteric & $819(51.9)$ & $33(55.0)$ & 786(51.8) & \\
\hline \multicolumn{5}{|l|}{ Comorbidities n (\%) } \\
\hline Hypertension & $794(50.3)$ & $44(73.3)$ & 752(49.6) & 0.000 \\
\hline prior stroke & $658(41.7)$ & 28(46.7) & $624(41.1)$ & 0.393 \\
\hline Diabetes & $371(23.5)$ & 21(35.0) & $352(23.2)$ & 0.035 \\
\hline Coronary heart disease & $465(29.5)$ & 14(23.3) & $450(29.7)$ & 0.291 \\
\hline Arrhythmia & $94(6.0)$ & $5(8.3)$ & $89(5.9)$ & 0.429 \\
\hline Pulmonary disease & $45(2.9)$ & $4(6.7)$ & $44(2.9)$ & 0.096 \\
\hline Tumor & $74(4.7)$ & $4(6.7)$ & $70(4.6)$ & 0.461 \\
\hline Comorbidities $\geq 3$ & $352(22.3)$ & $20(33.3)$ & 334(22.0) & 0.039 \\
\hline
\end{tabular}

\section{Risk Factors of AIS after Hip Fracture}

Table 5 shows the results of the multivariable analyses. In the whole sample, combined with hypertension (OR 2.743, 95\% Cl 1.471-5.115) and male sex(OR 1.946, 95\% Cl 1.146-3.304) were independently associated with AIS after hip fracture (Table 5). 
Table 5

Multivariate logistic regression for prediction of AIS after hip fracture

\begin{tabular}{|llll|}
\hline Model variables & OR & $95 \% \mathrm{Cl}$ & $\boldsymbol{p}$ Value \\
\hline Hypertension & 2.743 & $1.471-5.115$ & 0.001 \\
\hline Diabetes & 1.633 & $0.843-3.165$ & 0.146 \\
\hline Comorbidities $\geq 3$ & 0.906 & $0.450-1.825$ & 0.783 \\
\hline Male & 1.946 & $1.146-3.304$ & 0.014 \\
\hline Notes: $p<0.05$, statistical significance. & \\
\hline
\end{tabular}

\section{Discussion}

A total of $60(3.8 \%)$ older hip fracture patients occurred AIS in all 1577 cases. Among 60 AIS patients, the number of female was higher than that of male, but the incidence was higher in male than female. The most common cause of fall was limb weakness or instability. The most cerebral infarction type was PACl and the most infarction lesion was single cerebral infarction in 60 AIS patients. Most of them were located on the left side. $81.7 \%$ of AIS patients had mild (NIHSS 0-4) AIS. Older patients with AIS after hip fracture were more frequently complicated by hypertension, previous stroke, diabetes and were more likely to have hypoproteinemia, electrolyte imbalance, anemia, deep vein thrombosis, cardiac complications and pneumonia. Combined with hypertension and male sex were independent risk factors of the AIS after hip fracture in the elderly.

There were significantly more female among AIS cases in our study, which is due to the reason that female tend to live longer and are more likely to develop stroke and fracture in older people[16, 17]. The AIS incidence was higher in male than female in older fracture people in our study. Studies have shown that male sex is a significant risk factor for stroke[18], and the incidence of stroke in male before the age of 85 is higher than in female[19]. 75-84 years was the most common age group in our study. This may be due to the largest number of fracture patients in this age group. Fall is a major cause of fracture in older adults, and the most common fall-related injury was hip fracture[20].Elderly people often fall due to instability and weakness, which may be related to the decline of physical functions such as vision and movement, as well as the impairment of balance in the elderly[21]. The infarctions were solitary cerebral infarction and were located on the left side in our study. The result was consistent with previously published results of Hedna VS et al[22]. Left-sided strokes might be mentioned more often because they cause obvious symptoms like aphasia, while strokes on the right side can cause less obvious symptoms like hemineglector spatial disorientation[23]. We think that this can also be applied to patients with AIS after fracture.

The prevalence of AIS after hip fracture was 3.8\% in our study, which was consistent with prior study[24]. In our study, it was found that AIS patients after hip fracture were more frequently comorbid with chronic 
medical illness diseases, including hypertension, prior stroke and diabetes. Hypertension and diabetes are significant risk factors for the occurrence of cerebrovascular accidents (CVA) after hip fracture[25]. It has been observed that the carotid arteries of patients with diabetes are thicker and harder than those of the general population[26]. In addition, it has been reported that the carotid stiffness index of hypertensive patients is significantly higher than that of the general population[27]. Hypertension and diabetes accelerate the progression of atherosclerosis. Atherosclerosis is an important pathological cause of ischemic stroke. $30 \%$ of ischemic stroke are caused by carotid atherosclerosis[28]. The pathogenesis of ischemic stroke is considered to be arterial embolism caused by rupture of carotid plaque, ulceration, and platelet activation.

A study has shown that hip fracture is associated with increased risk of stroke[29]. Physical inactivity, fear and pain are common in the elderly hip fracture patients. In this case, the pre-existing cerebrovascular risk may be exacerbated. This may be associated with traumatic stress response. Fracture can lead to the release of stress hormones such as glucocorticoids, glucagon, epinephrine, thyroxine and others, which is called the "stress response"[30]. Stress response induces platelet aggregation and promotes microcirculation dysfunction and thrombosis[30]. A large number of inflammatory mediators caused by stress are released after hip fracture, and inflammation in the acute phase can affect thrombosis. Inflammation is usually related to oxidative stress. The strong oxidative activity can also destroy normal cell structure and promote the progress of cardiovascular and cerebrovascular diseases[31].

Patients with both fractures and strokes have a worse prognosis than those with single injury[32], and this may be related to their stronger traumatic stress response. Our observations showed that AIS patients after hip fractures suffered other adverse events such as hypoproteinemia, anemia, electrolyte disturbances, pneumonia, lower limb venous thrombosis and cardiovascular complications. Complications also affected each other, resulting in worse prognosis.

Hypoalbuminemia, electrolyte imbalance, and anemia were common complications after bone fracture in our study. Factors such as excessive blood loss and pain after the fracture induce stress response. Stress response puts the body in a state of enhanced catabolism and decreased anabolism, which may be related to the occurrence of hypoproteinemia and electrolyte disorder[33]. Stroke related disorders such as cognitive dysfunction, impaired consciousness, neurogenic vomiting, neurogenic dysphagia, and motor dysfunction also contribute to the development of hypoalbuminemia and electrolyte imbalance[34]. One study has shown that hypoalbuminemia occurs more frequently in patients with bone fracture and stroke and is associated with a poor prognosis[35]. Several studies have shown that human albumin therapy can combat thrombosis, stagnation and leukocyte adhesion of the microcirculation in the early stage of stroke, and provide neuroprotection for stroke patients[36, 37].

The traumatic stress response leads to the release of hormones such as catecholamines[38], which increase pulmonary artery systolic pressure and initiate pulmonary vasoconstriction and pulmonary interstitial edema. Moreover, an excessive stress response would impair organism immunity. These 
factors lead to the occurrence of pneumonia. In addition, swallowing difficulty, immune deficiency, nasogastric tube feeding, and poor oral hygiene from stroke can increase the risk of pneumonia. The occurrence of pneumonia increases the risk of in-hospital death[39]. In addition, after traumatic fracture, stress can activate the endogenous and exogenous coagulation system, resulting in hypercoagulability and coagulation dysfunction. This significantly increases the risk of deep vein thrombosis.

The persistent stress of older hip fracture patients can cause myocardial hypoxia and increase the imbalance between oxygen supply and myocardial demand. This is a common and complex pathophysiological mechanism that causes the occurrence of cardiac complications[40]. There are several common risk factors for stroke and ischemic heart disease. Underlying heart diseases, such as atrial fibrillation, valve defects, or congestive heart failure, increase the risk of stroke. Stroke interferes with autonomic control and can easily lead to cardiac complications in patients[41].Cardiac complications in patients with fracture or stroke can be fatal for the patients[42]. All the occurrence of these complications after hip fracture is associated with traumatic stress, and we need to strengthen the management of patients' breathing, circulation, blood, and thrombus to attenuate the stress response and improve the quality of patients' survival.

Furthermore, after we performed multivariate logistic regression on patients participated in this study, the data showed that comorbid with hypertension and male sex were significant risk factors for the AIS after hip fracture in the elderly. The mechanism of stroke caused by hypertension was discussed above. For this reason, we propose the following recommendations. Firstly, elderly patients comorbid with hypertension should prevent falls and fractures in their daily life. Secondly, Elderly patients comorbid with hypertension should pay attention to monitoring blood pressure, blood lipids, and preventing the occurrence and development of cerebrovascular disease. In addition, for these elderly patients who develop fracture, we should strengthen management to reduce the stress reaction and avoid the occurrence of stroke and other complications.

\section{Limitations}

There are some limitations to our study that warrant discussion. First, it is a retrospective nature of the study and the intrinsic limitation of design seems to be inescapable. Second, there is the possibility of selection bias owing to small sample size. The conclusions drawn cannot completely and reliably represent the general clinical characteristics. Third, patients with AIS after fracture surgery were not included in our study. Further study is needed to compare the different characteristics of AIS after hip fracture and AIS after surgery.

\section{Conclusions}

Elderly patients with AIS after hip fracture more often comorbid with hypertension, prior stoke and diabetes. Older patients who had hypertension are more likely to have AIS after hip fracture. AIS patients after hip fracture are prone to have multiple complications under traumatic stress, and we should 
enhance the management of these patients to reduce the stress response and avoid occurrence of complications.

\section{Abbreviations}

AIS: acute ischemic stroke; TACl: total anterior circulation infarction; PACl: partial anterior circulation infarction; POCl: posterior circulation infarction; LACl: lacunar infarction; NIHSS: National Institutes of Health stroke scale; CVA: cerebrovascular accidents

\section{Declarations}

\section{Acknowledgments}

None.

\section{Author contributions}

ZQ.W and ZY.H conceived the study. YQ.Z, MM.F, JF.G and YQ. Z supported study preparation and data collection. YQ.Z collected the data and drafted the manuscript. ZQ.W and ZY.H critically reviewed the manuscript for important intellectual content. All authors approved the final version of the manuscript.

\section{Funding}

The study was financially supported by the 2021 Hebei Provincial government funded the clinical medicine personnel training program

\section{Data Availability}

The data used to support the findings of this study are available from Zhiqian Wang upon request.

\section{Ethical approval and Informed consent}

This study was approved by the institutional review board of the third Hospital of Hebei Medical University in compliance with the Helsinki and an exemption from theinformed consent was obtained. All data were anonymized before the analysis to safeguard patient privacy.

\section{Consent for publication}

Not Applicable.

\section{Conflicts of interest}

The authors declare that there is no conflict of interest regarding the publication of this paper.

\section{References}


1. Zhang C, Feng J, Wang S, Gao P, Xu L, Zhu J, Jia J, Liu L, Liu G, Wang J et al: Incidence of and trends in hip fracture among adults in urban China: A nationwide retrospective cohort study. PLoS medicine 2020, 17(8):e1003180.

2. Schmidt AH, Braman JP, Duwelius PJ, McKee MD: Geriatric trauma: the role of immediate arthroplasty. The Journal of bone and joint surgery American volume 2013, 95(24):2230-2239.

3. Salhotra A, Shah HN, Levi B, Longaker MT: Mechanisms of bone development and repair. Nature reviews Molecular cell biology 2020, 21(11):696-711.

4. Veronese N, Maggi S: Epidemiology and social costs of hip fracture. Injury 2018, 49(8):1458-1460.

5. Hu F, Jiang C, Shen J, Tang P, Wang Y: Preoperative predictors for mortality following hip fracture surgery: a systematic review and meta-analysis. Injury 2012, 43(6):676-685.

6. Yu L, Zhu Y, Chen W, Bu H, Zhang Y: Incidence and risk factors associated with postoperative stroke in the elderly patients undergoing hip fracture surgery. Journal of orthopaedic surgery and research 2020, 15(1):429.

7. Zhang YW, Lu PP, Li YJ, Dai GC, Chen MH, Zhao YK, Cao MM, Rui YF: Prevalence, Characteristics, and Associated Risk Factors of the Elderly with Hip Fractures: A Cross-Sectional Analysis of NHANES 2005-2010. Clinical interventions in aging 2021, 16:177-185.

8. Samuel AM, Diaz-Collado PJ, Szolomayer LK, Nelson SJ, Webb ML, Lukasiewicz AM, Grauer JN: Incidence of and Risk Factors for Inpatient Stroke After Hip Fractures in the Elderly. Orthopedics 2018, 41(1):e27-e32.

9. Mathew RO, Hsu WH, Young Y: Effect of comorbidity on functional recovery after hip fracture in the elderly. American journal of physical medicine \& rehabilitation 2013, 92(8):686-696.

10. Rasouli MR, Tabatabaee RM, Maltenfort MG, Chen AF: Acute stroke after total joint arthroplasty: a population-based trend analysis. Journal of clinical anesthesia 2016, 34:15-20.

11. Bateman BT, Schumacher HC, Wang S, Shaefi S, Berman MF: Perioperative acute ischemic stroke in noncardiac and nonvascular surgery: incidence, risk factors, and outcomes. Anesthesiology 2009, 110(2):231-238.

12. Bijker JB, Persoon S, Peelen LM, Moons KG, Kalkman CJ, Kappelle LJ, van Klei WA: Intraoperative hypotension and perioperative ischemic stroke after general surgery: a nested case-control study. Anesthesiology 2012, 116(3):658-664.

13. Burgers PT, Hoogendoorn M, Van Woensel EA, Poolman RW, Bhandari M, Patka P, Van Lieshout EM: Total medical costs of treating femoral neck fracture patients with hemi- or total hip arthroplasty: a cost analysis of a multicenter prospective study. Osteoporosis international : a journal established as result of cooperation between the European Foundation for Osteoporosis and the National Osteoporosis Foundation of the USA 2016, 27(6):1999-2008.

14. Keel M, Trentz O: Pathophysiology of polytrauma. Injury 2005, 36(6):691-709.

15. Stroke--1989. Recommendations on stroke prevention, diagnosis, and therapy. Report of the WHO Task Force on Stroke and other Cerebrovascular Disorders. Stroke 1989, 20(10):1407-1431. 
16. Poupore N, Chosed R, Arce S, Rainer R, Goodwin RL, Nathaniel TI: Metabolomic Profiles of Men and Women Ischemic Stroke Patients. Diagnostics (Basel, Switzerland) 2021, 11(10).

17. Hannan MT, Broe KE, Cupples LA, Dufour AB, Rockwell M, Kiel DP: Height loss predicts subsequent hip fracture in men and women of the Framingham Study. Journal of bone and mineral research : the official journal of the American Society for Bone and Mineral Research 2012, 27(1):146-152.

18. Uchida M, Palmateer JM, Herson PS, DeVries AC, Cheng J, Hurn PD: Dose-dependent effects of androgens on outcome after focal cerebral ischemia in adult male mice. Journal of cerebral blood flow and metabolism : official journal of the International Society of Cerebral Blood Flow and Metabolism 2009, 29(8):1454-1462.

19. Petrea RE, Beiser AS, Seshadri S, Kelly-Hayes M, Kase CS, Wolf PA: Gender differences in stroke incidence and poststroke disability in the Framingham heart study. Stroke 2009, 40(4):1032-1037.

20. Moncada LVV, Mire LG: Preventing Falls in Older Persons. American family physician 2017, 96(4):240-247.

21. Ruiz-Ruiz L, Jimenez AR, Garcia-Villamil G, Seco F: Detecting Fall Risk and Frailty in Elders with Inertial Motion Sensors: A Survey of Significant Gait Parameters. Sensors (Basel, Switzerland) 2021, 21(20).

22. Hedna VS, Bodhit AN, Ansari S, Falchook AD, Stead L, Heilman KM, Waters MF: Hemispheric differences in ischemic stroke: is left-hemisphere stroke more common? Journal of clinical neurology (Seoul, Korea) 2013, 9(2):97-102.

23. Portegies ML, Selwaness M, Hofman A, Koudstaal PJ, Vernooij MW, Ikram MA: Left-sided strokes are more often recognized than right-sided strokes: the Rotterdam study. Stroke 2015, 46(1):252-254.

24. An Q, Chen Z, Huo K, Su H, Qu QM: Risk factors for ischemic stroke post bone fracture. Journal of clinical neuroscience : official journal of the Neurosurgical Society of Australasia 2019, 59:224-228.

25. Atzmon R, Sharfman ZT, Efrati N, Shohat N, Brin Y, Hetsroni I, Nyska M, Palmanovich E: Cerebrovascular accidents associated with hip fractures: morbidity and mortality-5-year survival. J Orthop Surg Res 2018, 13(1):161.

26. Matsumoto K, Sera Y, Nakamura H, Ueki Y, Miyake S: Correlation between common carotid arterial wall thickness and ischemic stroke in patients with type 2 diabetes mellitus. Metabolism: clinical and experimental 2002, 51(2):244-247.

27. Giannarelli C, Bianchini E, Bruno RM, Magagna A, Landini L, Faita F, Gemignani V, Penno G, Taddei S, Ghiadoni L: Local carotid stiffness and intima-media thickness assessment by a novel ultrasoundbased system in essential hypertension. Atherosclerosis 2012, 223(2):372-377.

28. Crombag G, van Hoof RHM, Holtackers RJ, Schreuder F, Truijman MTB, Schreuder T, van Orshoven NP, Mess WH, Hofman PAM, van Oostenbrugge RJ et al: Symptomatic Carotid Plaques Demonstrate Less Leaky Plaque Microvasculature Compared With the Contralateral Side: A Dynamic ContrastEnhanced Magnetic Resonance Imaging Study. Journal of the American Heart Association 2019, 8(8):e011832. 
29. Kang JH, Chung SD, Xirasagar S, Jaw FS, Lin HC: Increased risk of stroke in the year after a hip fracture: a population-based follow-up study. Stroke 2011, 42(2):336-341.

30. Chen Y, Yang X, Meng K, Zeng Z, Ma B, Liu X, Qi B, Cui S, Cao P, Yang Y: Stress-induced hyperglycemia after hip fracture and the increased risk of acute myocardial infarction in nondiabetic patients. Diabetes care 2013, 36(10):3328-3332.

31. Su JH, Luo MY, Liang N, Gong SX, Chen W, Huang WQ, Tian Y, Wang AP: Interleukin-6: A Novel Target for Cardio-Cerebrovascular Diseases. Frontiers in pharmacology 2021, 12:745061.

32. Wei M, Lyu H, Huo K, Su H: Impact of Bone Fracture on Ischemic Stroke Recovery. International journal of molecular sciences 2018, 19(5).

33. Carpintero P, Caeiro JR, Carpintero R, Morales A, Silva S, Mesa M: Complications of hip fractures: A review. World journal of orthopedics 2014, 5(4):402-411.

34. Chen YD, Li SJ, Sun FH, Liu YY, Hu WL: Monitoring of medical complications after acute ischemic stroke in a neurological intensive care unit. European neurology 2011, 66(4):204-209.

35. Wilson JM, Lunati MP, Grabel ZJ, Staley CA, Schwartz AM, Schenker ML: Hypoalbuminemia Is an Independent Risk Factor for 30-Day Mortality, Postoperative Complications, Readmission, and Reoperation in the Operative Lower Extremity Orthopaedic Trauma Patient. Journal of orthopaedic trauma 2019, 33(6):284-291.

36. Belayev L, Liu Y, Zhao W, Busto R, Ginsberg MD: Human albumin therapy of acute ischemic stroke: marked neuroprotective efficacy at moderate doses and with a broad therapeutic window. Stroke 2001, 32(2):553-560.

37. Belayev L, Busto R, Zhao W, Clemens JA, Ginsberg MD: Effect of delayed albumin hemodilution on infarction volume and brain edema after transient middle cerebral artery occlusion in rats. Journal of neurosurgery 1997, 87(4):595-601.

38. Hašková P, Koubková L, Vávrová A, Macková E, Hrušková K, Kovaříková P, Vávrová K, Simůnek T: Comparison of various iron chelators used in clinical practice as protecting agents against catecholamine-induced oxidative injury and cardiotoxicity. Toxicology 2011, 289(2-3):122-131.

39. Chen Y, Yang H, Wei H, Chen Y, Lan M: Stroke-associated pneumonia: A bibliometric analysis of worldwide trends from 2003 to 2020. Medicine 2021, 100(38):e27321.

40. Fu M, Wang Z, Liu Y: Effects of Xinkeshu combined with levosimendan on perioperative heart failure in oldest-old patients with hip fractures. Journal of traditional Chinese medicine $=$ Chung $i$ tsa chih ying wen pan 2020, 40(5):870-874.

41. Baranchuk A, Nault MA, Morillo CA: The central nervous system and sudden cardiac death: what should we know? Cardiology journal 2009, 16(2):105-112.

42. Prosser J, MacGregor L, Lees KR, Diener HC, Hacke W, Davis S: Predictors of early cardiac morbidity and mortality after ischemic stroke. Stroke 2007, 38(8):2295-2302.

\section{Figures}




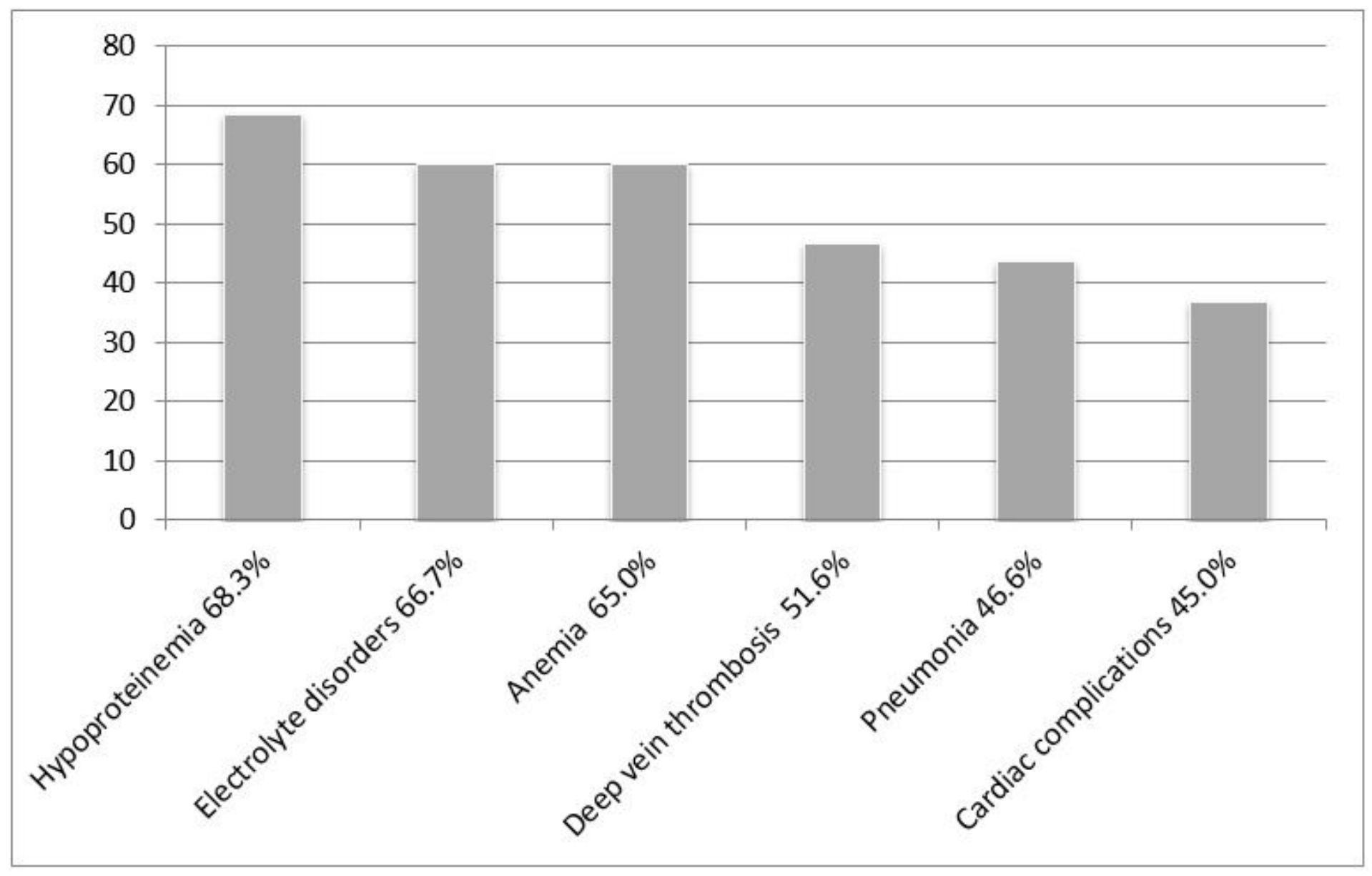

Figure 1

The incidence of other complications 